\title{
Trayectorias de vida y elección profesional: elementos influyentes en la elección profesional de estudiantes de psicología, sociología y trabajo social
}

\author{
Nicole Salas Salazar* \\ Carolina Carrasco González* \\ Angie Flores Garay* \\ Daniela León Urrutia*
}

\section{RESUMEN}

\begin{abstract}
El presente artículo señala los resultados de una investigación correspondiente a un estudio cualitativo-interpretativo, que describe los elementos comunes y diferenciadores en las trayectorias vitales de estudiantes de psicología, sociología y trabajo social, relacionados con su opción de formación profesional. Teniendo en consideración el papel desvalorizado de las disciplinas de las ciencias sociales en el contexto socioeconómico actual y la obtención de bajos salarios de estas profesiones en el campo laboral, se problematiza la idea de que aun así, sigue siendo de las áreas más demandadas por los estudiantes al momento de su ingreso a la educación superior, en ello radica la importancia de conocer los elementos en las trayectorias de vida de los estudiantes que inciden en su elección profesional al optar por el área de las ciencias sociales y por las diferentes profesiones en particular.
\end{abstract}

Palabras Clave: Trayectorias de vida - Ciencias sociales - Formación profesional - Vocación profesional

Trajetórias de vida e eleição profissional: elementos que influenciam a eleição profissional de estudantes de psicologia, sociologia e trabalho social

RESUMO

O presente artigo indica os resultados de uma investigação correspondente a um estudo cualitativo-interpretativo, que descreve os elementos comuns e diferenciadores nas trajetórias vitais de estudantes de psicologia,

* Chilenas. Estudiantes de la Escuela de Trabajo Social de la Universidad Católica Silva Henríquez. Autoras del seminario para optar al grado de licenciado en trabajo social titulado "Trayectorias de vida y elección profesional: elementos influyentes en la elección profesional de estudiantes de psicología, sociología y trabajo social", guiado por la profesora Sandra Iturrieta Olivares, que da origen al presente artículo. Correo electrónico: nicolef.salass@hotmail.com 
Trayectorias de vida y elección profesional: elementos influyentes en la elección profesional de estudiantes de psicología, sociología y trabajo social / Nicole Salas Salazar; Carolina Carrasco González; Angie Flores Garay; Daniela León Urrutia

sociologia e trabalho social, relacionados com sua opção de formação profissional. Tendo em consideração o papel desvalorizado das disciplinas das ciências sociais no contexto socioeconômico atual e a obtenção dos baixos salários destas profissões no campo laboral, problematiza-se a ideia de que ainda assim, continuam sendo as disciplinas mais demandadas pelos estudantes ao momento de seu ingresso na educação superior. Nisto radica a importância de conhecer os elementos nas trajetórias de vida dos estudantes que incidem em sua eleição profissional ao optar pelo campo das ciências sociais e pelo as diferentes profissões em particular.

Palavras Chave: Trajetórias de vida - Ciências sociais - Formação profissional - Vocação profissional

\title{
Trajectories of life and professional training choice: influential elements in professional training choice of psychology, sociology and social work students
}

\begin{abstract}
This article outlines the results of a qualitative-interpretive study, which describes the commonalities and differentiators in the life trajectories of students of psychology, sociology and social work related to their professional training options. Considering the devalued role of social science disciplines in the current economic context and low wages of related-field professions in the workplace, it is argued that they still remain the most preferred options among students at the time of starting their higher education studies. Hence it is critical to identify the elements in the life trajectories of students that influence their professional training choice of the social sciences disciplines and professions.
\end{abstract}

Keywords: Trajectories of Life - Social Sciences - Professional Training Professional Vocation

\section{Antecedentes}

Las Ciencias Sociales en América Latina han experimentado cambios a finales del siglo XX originadas entre otros factores en el fracaso del poder centralizado, principalmente debido a las reformulaciones en la noción de Estado, siendo una evidencia de ello los procesos de liberalización económica desarrollados en la región durante los regímenes dictatoriales, donde se comenzó a priorizar programas de estabilización macroeconómica que obligaba a los Estados a reducir el gasto público y a someterlo al desempeño de la política monetaria (Cortés, 1999: 24). De esta manera, se ve limitado el accionar de las Ciencias Sociales en la región, donde a diferencia de tiempos anteriores, se han visto subyugadas al sistema económico imperante. 
En el caso de Chile la situación no varía mucho, ya que el sistema económico neoliberal se instauró de la misma manera, y es en aquel contexto dictatorial donde es posible identificar la reducción a la mínima expresión, de la intervención estatal en materias económicas y sociales (mediante las privatizaciones de servicios que hasta el momento eran públicos), lo que genera, a su vez, que las profesiones de las Ciencias Sociales comiencen a verse afectadas en la valoración o estatus social que poseen, puesto que los principios profesionales de éstas no serían coherentes con tales estándares que dan vida al modelo mencionado (patrones de consumo, oferta/demanda, rentabilidad, etcétera).

Por otra parte, a principios de los años sesenta en Chile se logró la consolidación de las identidades disciplinarias de las Ciencias Sociales, ligada a la formación e investigación científica. En dicha época, se aprecia un momento expansivo de éstas, alcanzando un cierto prestigio y una demanda social que proviene de sus conocimientos (Garretón, 2005: 6), cuestión que se desvanece unas décadas más tarde.

En el escenario antes descrito es que se contextualiza la investigación que da origen al presente artículo, del que fueron parte estudiantes de algunas de las profesiones que componen el área de las Ciencias Sociales, y que a su vez convergen en las intervenciones sociales directas e indirectas (Miranda, 2003: 172). De modo que la muestra quedó conformada por estudiantes de psicología, sociología y trabajo social.

Es posible constatar, según datos de Futuro Laboral (2010), que las Ciencias Sociales se encuentran dentro de las cuatro áreas más demandadas por los estudiantes que hacen ingreso a la educación superior en primer año, ello no obstante que las remuneraciones de las mismas evidencian bajos salarios, siendo en promedio mensual de $\$ 497.815^{1}$, cuestión que tiende a reflejar lo expresado sobre la valoración social de estas profesiones.

A este contexto se adiciona por una parte, la expansión de estas profesiones y la diversificación de las Ciencias Sociales, planteándose así una incógnita sobre la especificidad profesional (Garretón, 2005: 32). En relación a ello se "suele sostener que el trabajo interdisciplinario es una modalidad cada vez más requerida y necesaria para abordar las problemáticas de la

1 Empleabilidad en primer año (2009). Cohorte de titulados en 2007 y 2008 (Fuente: Futuro Laboral, 2010). 
Trayectorias de vida y elección profesional: elementos influyentes en la elección profesional de estudiantes de psicología, sociología y trabajo social / Nicole Salas Salazar; Carolina Carrasco González; Angie Flores Garay; Daniela León Urrutia

sociedad" (Chacón et al., 2004: 11). Pero más allá de ello es posible sostener que las tres profesiones estudiadas: Psicología, sociología y trabajo social, presentarían límites profesionales superpuestos en el ejercicio laboral, puesto que los profesionales de las ciencias sociales comparten metodologías, objetos, teorías, etcétera, diferenciándose, sin embargo, en la mirada que cada una de éstas le da a la realidad social y a los problemas sociales (Araya et al. 2009: 35).

\section{Trayectorias de vida y elección profesional}

Si consideramos que las trayectorias vitales se organizan "a partir de dos conceptos centrales: trayectoria y transición. El primero hace referencia al itinerario de vida de los sujetos; el proceso que marca el comienzo y el fin de un ciclo de vida entendido como un todo unitario, mientras que el segundo hace referencia a los diversos episodios en que se desagrega esa trayectoria, no necesariamente predefinidos o predeterminados, pero que marcan cambios en el estado, posición o situación de los individuos al interior de la sociedad." (Sepúlveda 2010:34). Y si además tenemos en cuenta que la elección profesional es una decisión que se forja a partir de las vivencias de los propios sujetos, es que las trayectorias de vida podrían determinarla ya que las trayectorias vitales están constituidas por un conjunto de elementos individuales y colectivos. En tal sentido, Mettifogo y Sepúlveda (2005: 18) sostienen que el análisis de las trayectorias de vida permite analizar y comprender el sentido que las personas otorgan a sus actos, las lógicas con que organizan su vida cotidiana, sus sistemas de vinculaciones con otros y los principios que sustentan sus prácticas.

En la investigación en ciencias sociales la utilización de las trayectorias de vida, han mostrado importantes desarrollos, permitiendo articular significados subjetivos de experiencias y prácticas sociales (Cornejo et al. 2008: 29). Junto con lo anterior, es de importancia sostener que la investigación a través de las trayectorias de vida no busca dar una ilusión de verdad o certeza, sino que acepta la incertidumbre e impredictibilidad de la vida, sin pretender que seamos seres epistemológicamente objetivos cuando somos ontológicamente subjetivos. Se trata, finalmente, de acercarse a un sujeto complejo (Cornejo et al. 2008).

En consecuencia, las trayectorias de vida se constituyen en una manera apropiada para conocer los elementos influyentes, 
comunes y diferenciadores en la vida de los estudiantes para su posterior elección profesional, centrando la mirada en las historias y vivencias que ellos mismos distinguen como desencadenantes de dicha decisión, lo que es posible de identificar a partir de los hitos y transiciones presentes en los relatos de los estudiantes.

\section{Metodología}

Este estudio se realizó bajo el método cualitativo-interpretativo, empleando relatos de vida como técnica de recolección de datos. Los informantes fueron estudiantes regulares de las profesiones antes mencionadas, de universidades pertenecientes y no pertenecientes al Consejo de Rectores de Chile.

Se propuso entrevistar al menos un estudiante de cada universidad, hasta que la información fuese saturada, agregando además como criterio de rigurosidad la validez intersubjetiva. Cabe mencionar que se realizó un total de diecinueve entrevistas a estudiantes de las profesiones estudiadas, donde ocho de éstas correspondieron a Psicología, tres a Sociología y ocho a Trabajo Social.

El trabajo con los datos consistió en análisis categorial simple, a través del que fue posible establecer las categorías y subcategorías sobre las cuales se articulan los discursos de los estudiantes entrevistados.

\section{Resultados del estudio}

Luego de realizado el trabajo de campo, fue posible identificar tres grandes categorías con sus respectivas sub-categorías, en el relato de los estudiantes, que los llevarían a explicar la forma en la que desarrollan y consolidan sus respectivas elecciones profesionales.

La primera corresponde a ciertos "hitos que gatillan la elección"; esta categoría hace alusión a los eventos significativos dentro de la trayectoria de vida de los estudiantes entrevistados, que ellos consideran como determinantes en su elección profesional²:

2 Subcategorías "Trabajo de las madres" e "Iglesia" son emergentes de Trabajo Social; subcategoría "Profesores" son emergentes en Psicología y Sociología. 
Trayectorias de vida y elección profesional: elementos influyentes en la elección profesional de estudiantes de psicología, sociología y trabajo social / Nicole Salas Salazar; Carolina Carrasco González; Angie Flores Garay; Daniela León Urrutia

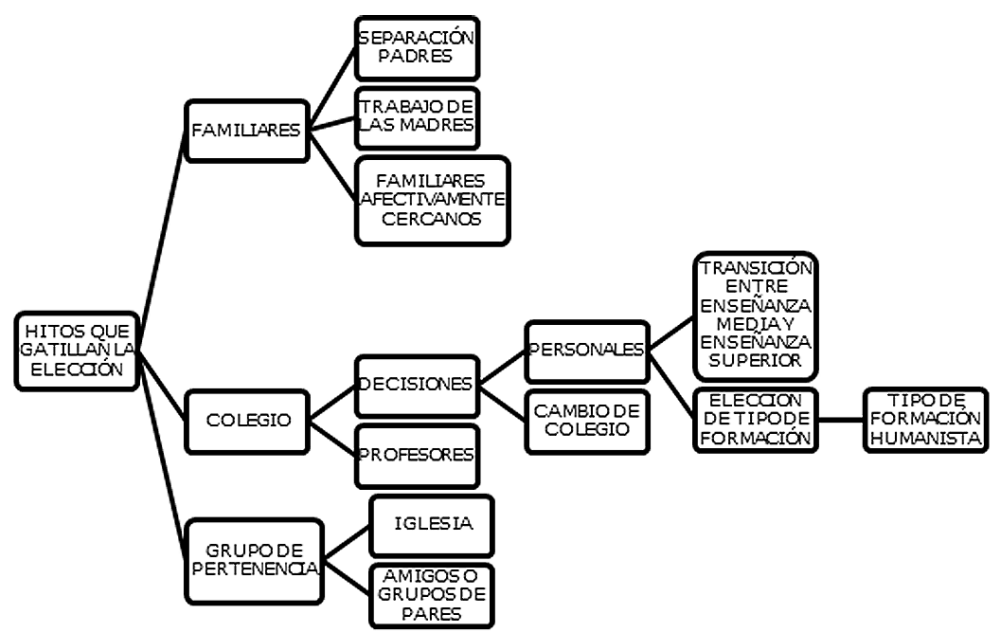

La segunda categoría se refiere al "tipo de elección de la profesión". Esta categoría pretende dar a conocer la forma en la cual se escogió una profesión específica:

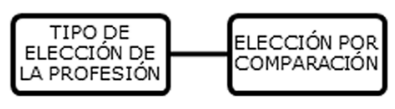

Y la última categoría está compuesta por aquellas “características personales auto-atribuidas", la cual da a conocer diversos atributos auto-atribuidos por los estudiantes entrevistados, que consideran influyentes en la elección profesional efectuada:

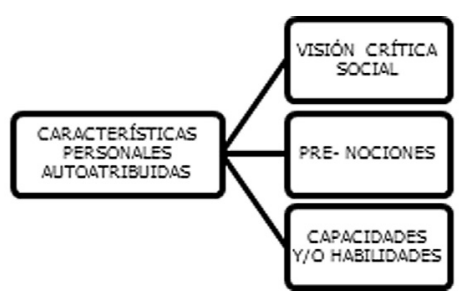

Como es posible observar en los esquemas precedentes, cada categoría fue subcategorizada hasta llegar a establecer los elementos que finalmente han sido influyentes, en la elección del área de las ciencias sociales y de profesión específica. A continuación se resumen los principales resultados alcanzados y las conclusiones más transcendentales a las que ha sido posible arribar en este estudio. 


\section{Conclusiones}

Sobre la base de las categorías anteriormente mencionadas ha sido posible identificar elementos comunes e influyentes en la elección profesional, en que las transiciones más importantes de los estudiantes tienen relación con aspectos vividos principalmente en dos esferas: la familia y las experiencias vivenciadas durante la permanencia en la enseñanza secundaria. Estos, son dos momentos cruciales y desencadenantes de la elección futura, tanto del área de las ciencias sociales, como también de una profesión en particular.

En el caso de la elección del área de las ciencias sociales, tal opción estaría influida por experiencia positivas vividas en la enseñanza secundaria respecto del área y también por las características personales auto-atribuidas por los estudiantes, tales como una mayor afinidad en asignaturas como historia y lenguaje en el caso de trabajo social y sociología, y de filosofía en el caso de psicología, lo que los guía hacia la elección por el área humanista. En un primer momento, los estudiantes vinculan dichas asignaturas con el área de las ciencias sociales. Mientras que en un segundo momento se detienen a analizar las profesiones que de ella se desprenden. Por consiguiente, la transición de mayor relevancia en este aspecto sería el paso de segundo a tercer año de enseñanza media, como un momento crucial en la futura elección.

Por otra parte, dentro de las trayectorias vitales analizadas, también ha sido posible identificar elementos diferenciadores en la elección profesional, donde fue posible distinguir tres grandes líneas influyentes en la elección de una profesión específica:

Influencia de familiares afectivamente cercanos: Dentro de este punto es posible visualizar a familiares que son profesionales o se encuentran cursando estudios en alguna de las profesiones del área de las ciencias sociales o, por el contario, son simplemente influyentes en la apertura de mundo, lo que repercute a su vez en la conformación de una conciencia crítica por parte de los estudiantes, sin que necesariamente sean parte de esta área.

Sin embargo, a pesar de que ciertos familiares cercanos sean influyentes en la elección profesional, ya sea a través de experiencias vividas con ellos, o por la transmisión de conocimientos y/o pensamientos y gustos, también la familia juega un rol cuestionador en dicha decisión, lo cual estaría 
Trayectorias de vida y elección profesional: elementos influyentes en la elección profesional de estudiantes de psicología, sociología y trabajo social / Nicole Salas Salazar; Carolina Carrasco González; Angie Flores Garay; Daniela León Urrutia

vinculado a la poca valorización social que tienen en la actualidad las profesiones de las ciencias sociales estudiadas.

Desconocimiento del quehacer profesional: este desconocimiento se da en mayor medida en las profesiones de trabajo social y sociología, donde los estudiantes escogen una profesión en base a la afinidad que tienen con el área social en su conjunto y también por las pre-nociones que se posee de cada una de ellas, la cual muchas veces está determinada por la asistencia a ferias universitarias. Cabe resaltar que el desconocimiento sobre estas profesiones nos puede alentar a desarrollar una reflexión sobre la visibilidad del quehacer profesional, y del mismo modo, sobre los límites difusos entre dichos quehaceres; ello nos insta a reflexionar sobre la manera en que estas profesiones se posicionan y se diferencian, en un contexto social complejo.

Sumado a lo anterior, es posible sostener también, que el hecho de que el primer acercamiento a las profesiones sea mediante la asistencia a ferias universitarias, corre el riesgo de no lograr dar cabida a los supuestos teóricos que las sustentan y tampoco vislumbrar el ejercicio profesional específico, lo cual puede conllevar a formarse ideas equívocas sobre la profesión que se está eligiendo.

Idea de ayuda: en lo que respecta a las prenociones, éstas se encuentran enmarcadas por el concepto de ayuda. Dentro de ella, resulta relevante destacar que esta idea se establece como otra determinante en la elección, presentándose sin embargo, distintas visiones para concebirla según la profesión específica:

- Psicología: a diferencia de las otras dos profesiones, se visualiza un énfasis en la solución de problemas de los otros como un mecanismo para la solución de conflictos propios. Es decir, en gran medida la elección de esta profesión es guiada por las vivencias personales que han influido en forma significativa en sus vidas, surgiendo su deseo por ayudar a otro que haya experimentado situaciones similares a las vivenciadas. En el caso de Psicología, la visión de los problemas sociales y de la forma de cómo abordarlos, está más centrada en el individuo como ente aislado del medio sociocultural, respecto de las otras dos profesiones. Por otra parte, los estudiantes de esta profesión tienen en común el interés por analizar, observar, escuchar y aconsejar a los demás, el ser empáticos y la satisfacción de poder orientar a los otros. Frente a esto, se puede mencionar que la noción de ayuda de los entrevistados radica en aportar 
al cambio social, a partir de la modificación en la mentalidad del ser humano y, a su vez, lograr entender las reacciones de las personas y dar respuesta a diversas problemáticas sociales a partir de una perspectiva centrada en el individuo, potenciando de ese modo la transformación social.

- Sociología: los entrevistados ponen un mayor énfasis, respecto de las otras dos profesiones, en el análisis de los problemas sociales, teniendo una visión más distante o periférica de los mismos, donde la idea de ayuda para los estudiantes de sociología, surge a raíz del énfasis puesto en el análisis crítico de la realidad actual, en que actuarían para transformarla, comprenderla y analizarla, contribuyendo a superar situaciones adversas mediante la aplicación de los conocimientos de bienestar social y de desarrollo social. A ello, se agrega la necesidad personal de investigar, conocer y comprender en mayor profundidad los procesos sociales, para así potenciar cambios a nivel macro social. Asimismo, se evidencia en sociología y trabajo social, una visión social del concepto de ayuda, puesto que se sustenta en ideas de injusticia, desigualdad, derechos, etcétera, cuestión que no es mencionada por los estudiantes de psicología.

- Trabajo Social: los estudiantes de esta profesión otorgan mayor énfasis a la solución de los problemas sociales; en este sentido, éstos se sienten partícipes en la solución de los mismos. Además, se puede sostener que los estudiantes eligen esta profesión al constatar mediante sus propias vivencias las injusticias sociales, lo cual determina su descontento con las desigualdades sociales y su deseo de generar un cambio. Junto a lo anterior, también influye en la elección profesional, la participación en distintas actividades solidarias realizadas a través de instituciones como la Iglesia, donde la visión de diferentes realidades configura la aspiración por ser un profesional que tenga contacto directo con las personas. Por consiguiente, los estudiantes de trabajo social escogerían la profesión debido a que fueron testigos de ciertas problemáticas o vulnerabilidades sociales a lo largo de sus trayectorias de vida, constituyéndose así en un hito relevante para la cohorte estudiada.

En síntesis, el aspecto descrito es uno de los elementos comunes, pero al mismo tiempo diferenciador, más importante a la hora de responder sobre el porqué de la elección profesional. 
Trayectorias de vida y elección profesional: elementos influyentes en la elección profesional de estudiantes de psicología, sociología y trabajo social / Nicole Salas Salazar; Carolina Carrasco González; Angie Flores Garay; Daniela León Urrutia

Por otro lado, existen dos categorías emergentes identificadas en la profesión de trabajo social. En primer lugar el trabajo de las madres, donde podemos concluir que la figura materna influyó en la elección por parte de los estudiantes de esta profesión, catalogada tradicionalmente como una carrera femenina, cuestión que no es posible identificar en las otras dos profesiones estudiadas, debido a que las mujeres son asociadas culturalmente con el rol de la ayuda, lo cual converge con una visión asistencialista, aún predominante en el ejercicio profesional del trabajo social.

La segunda categoría emergente en trabajo social es la referida al grupo de pertenencia, específicamente a la vinculación con la Iglesia Católica, donde ésta fortalece la necesidad de ayudar a los demás y genera cierta inquietud social, además de presentarse como una experiencia donde se conocen distintas realidades y se concientiza de las desigualdades sociales.

Finalmente, es posible establecer la necesidad de reflexionar sobre la relación entre la formación académica, el mercado laboral y los límites difusos existentes en el quehacer profesional de las disciplinas estudiadas, puesto que la sobrevaloración de conceptos como por ejemplo, profesional integral, interdisciplinariedad o multidisciplinariedad superaría, muchas veces, el interés por restablecer el debate sobre la importancia de las especificidades en el ejercicio profesional, por lo tanto, se consideraría importante tener presente esta sugerencia al momento de la formación académica.

Para finalizar, cabría hacer alusión a que el estudio realizado deja abiertas algunas interrogantes referidas a, por ejemplo: qué sucede con los elementos diferenciadores forjados a lo largo de las trayectorias vitales, una vez que los estudiantes ingresan a estudiar las respectivas profesiones; ¿son estos considerados para el logro de aprendizajes significativos?

\section{Referencias bibliográficas}

ARAYA C.; ARAYA C.; CATALÁN T.; MORGADO C.; SOTO N.; VÁSQUEZ N. (2009). Empleabilidad de sociólogos y antropólogos: una descripción comparativa con trabajo social. Seminario de Grado. Docente guía: Sandra Iturrieta Olivares. Universidad Católica Silva Henríquez, Santiago, Chile. 
CORTÉS P. (1999). La política social en América Latina: Notas para su evaluación al final del milenio. Revista Perspectivas (8). Departamento de Trabajo Social. Universidad Católica Silva Henríquez. Santiago, Chile.

CHACÓN C., GONZÁLEZ A., ISLA A., RIVERA F. Y VEJAR V. (2004). “El imaginario social de equipos interdisciplinarios y significación del quehacer del Trabajador Social". Tesis para optar al Título de Asistente Social, Licenciado en Desarrollo Familiar y Social. Docente guía: Magaly Cabrolié Vargas. Universidad Católica de Temuco. Temuco, Chile. Recuperado en abril 2011: http://biblioteca.uct. cl/tesis/carolina-chacon-alejandra-gonzalezalicia-isla-francisca-rivera-viviana-vejar/tesis. pdf

CORNEJO M., MENDOZA F. Y ROJAS R. (2008). La Investigación con Relatos de Vida: Pistas y Opciones del Diseño Metodológico. Pontificia Universidad Católica de Chile. Psykhe, 17 (1), p. 29-39. Recuperado en agosto 2011: http://www.scielo.cl/pdf/psykhe/ v17n1/art04.pdf

FUTURO LABORAL (2010). Base de Datos (XLS). Recuperado en mayo 2011: http://www.futurolaboral.cl/

GARRETÓN M. (2005). Las Ciencias Sociales en Chile. Institucionalización, ruptura y renacimiento. Versión en castellano del artículo "Social Sciences and society in Chile: institutionalization, breakdown and rebirth in Chile" a publicarse en la traducción de Social Sciences in Latin America. Special Issue del volumen 44, Nos 2-3, Junio Septiembre 2005 de Social Sciences Information sur les Sciences Sociales (SagePublications), por Siglo XXI, México. Recuperado en marzo de 2011: http:// www.insumisos.com/lecturasinsumisas/ Ciencias\%20sociales\%20en\%20Chile.pdf

METTIFOGO D. Y SEPÚlVEDA R. (2005). Trayectorias de Vida de jóvenes infractores de ley. Centros de estudios de seguridad ciudadana (CESC), Universidad de Chile, Instituto de asuntos públicos. Santiago, Chile. Recuperado en abril 2011: http://www. unifr.ch/ddp1/derechopenal/obrasportales/ op_20080612_43.pdf

MIRANDA M. (2003). Pragmatismo, Interaccionismo simbólico y Trabajo Social. De cómo la caridad y la 
Trayectorias de vida y elección profesional: elementos influyentes en la elección profesional de estudiantes de psicología, sociología y trabajo social / Nicole Salas Salazar; Carolina Carrasco González; Angie Flores Garay; Daniela León Urrutia

filantropía se hicieron científicas. Tesis doctoral, Universitat Rovira I Virgili. Director de la Tesis Josep $\mathrm{M}^{\mathrm{a}}$ Comelles, Tarragona, España. Recuperado en marzo 2011: http://tdx.cat/ bitstream/handle/10803/8406/tesis_completa. pdf?sequence $=22$

Sepúlveda, L. (2010). Las trayectorias y el análisis de curso de vida como fuentes de conocimiento y orientación de políticas sociales. Revista de Trabajo Social Perspectivas. Año XV. N. 21. Escuela de Trabajo Social. Universidad Católica Silva Henríquez. Santiago de Chile. 